\title{
A NOVEL ARCHITECTURAL FRAMEWORK FOR AGGREGATED SUBSCRIBER PROFILE
}

\author{
${ }^{1}$ Jagannadham Dulipala and ${ }^{2} \mathbf{P}$. Seethalakshmi \\ ${ }^{1}$ Anna University, BIT Campus Tiruchirappalli, India, 620024, India \\ ${ }^{2}$ Department of ECE, Anna University, BIT Campus Tiruchirappalli, India, 620024, India
}

Received 2013-10-18; Revised 2013-10-18; Accepted 2013-11-29

\begin{abstract}
Subscriber/User's data in a Communication Service Provider's (CSP) Environment spread across different "silo type" Network Elements (subscriber data sources) and systems such as Customer Relationship Management System (CRM), Home Location Register/Visitor Location Register (HLR/VLR), Home Subscriber Server (HSS), Policy Charging Rules Function (PCRF), Business Support System/Operation Support System (BSS/OSS). The proliferation of convergent telecom applications and value added services necessitates CSPs to be equipped with unified subscriber data management systems to enable Application Service Providers and Enterprises to build and offer subscriber oriented applications and services. Rapid service development warrants the need for general inter-faces for subscriber data access rather than multiple traditional data access interfaces and associated complexities. The Proposed Aggregated Subscriber Profile Architectural Framework abstracts the Network Elements and integration complexities by providing aggregated profile to 3rd party applications. The proposed Architectural Framework is based on Data Oriented Architecture (DOA), Service Oriented Architecture principles. Proof of concept implementation details along with performance results for a typical aggregated data model defined based on previous experiences, discussed.
\end{abstract}

Keywords: Service Oriented Architecture, Data Services, Communication Service Providers, Subscriber Data Management, Data Aggregation

\section{INTRODUCTION}

Communication is all about connecting with others. Humans thrust to participate in communities of their interests The Internet and Social Networks solved these fundamental needs and succeeded. Communication Service Providers (CSPs) are exploring all possible opportunities and ways to offer more than just providing connectivity, network and telephony services to their subscribers (Lucent, 2011). CSPs for all their strategic and converged service offerings need to achieve dynamic subscriber context and profile which requires integration of their Service Delivery Infrastructure with external data sources including Social Media. On the other hand, Social Media and Internet companies are interested to integrate with various Communication Service Providers for their premium services and application billing needs. Hence to roll out such convergent Services and offerings, extended aggregated profile information of their subscribers is necessary. The proposed Architecture is extendable to integrate with any external data sources through corresponding data adapters to achieve the unified profile. CSPs espouse Service Delivery Platform (SDP) and Infrastructure, Middleware Infrastructure which essentially help them to expose network capabilities as Service Enablers and address the needs of converged applications. While SDP provides proliferated convergent Telecom IT services and applications as described by (Ohnishi et al., 2007), accessing subscriber profile in an Operator's environment is still a challenge with silo type data sources or network elements. Convergent Telecom applications and services require a robust unified

Corresponding Author: Jagannadham Dulipala, Anna University, BIT Campus Tiruchirappalli, India, 620024, India 
subscriber data access and management mechanisms. Hence to roll out such convergent Services and offerings, aggregated subscriber profile is an obvious requirement.

\section{RELATED WORK}

Subscriber profile from various service providers is aggregated to provide an aggregate subscriber profile as stated by (Karvell et al., 2011). This study explains about an aggregated subscriber profile that can be generated irrespective of data formats, conflicts, underlying data source servers and protocols/interfaces of any kind.

A good attempt for managing a telecommunications network comprises of Network Management System (NMS) and log-on credentials for authentication. For realization of this with better management scheme, (Perry et al., 2006) proposed log-on credential system for authentication. This study highlights the subscriber profile management system in a more reliable manner. In contrast, the system found from its analysis that it lags in session file management system. The authors showed the solution in a way that creates a remote file invocation system. An Enterprise Modeling framework as proposed by (Chan, 2004) for deployment of data warehouses emphasizes the separation of the conceptual data model from the physical data models in an enterprise. The work shows solutions for enterprise operations in an analytical manner. The virtual construct of this enterprise model provides the insulation of the enterprise from process and technical abilities such as scale and scope for reliable methodology. The integrated CRM introduced by (Chan, 2005) transacts the data in conjunction with legacy and external data extraction. For an enterprise data model, ETL based approach could be a better solution, however it is understood that the same could not be a viable solution for Telecom Service Providers based on huge volume of data explained by (Adzic et al., 2001).

Various Telecom Equipment Manufacturers exploring and addressing subscriber data management solutions such as SingleSDB stated by (Yuhong, 2011), CUDB and UPG solution published by (Ericsson, 2011) addressing through various proprietary solutions along with their Network Elements offerings. Research by (Mottishaw, 2009) reported a detailed study about Subscriber Data Management (SDM) Solution. In this report, Authors explained about contemporary SDM Solutions and challenges. Authors reported that CSPs managing subscriber data in different silos of SDM elements and support distinct operational needs such as multiple network authentication systems, customer care systems. The analysis of this study signifies in bringing third parties and value chains in delivering brand new and innovative services. Authors suggested various deployment scenarios and recommended that SDM platform of extending cross network consolidation with a north bound access to subscriber data and support 3rd party access to data.

Based on the above literature, in this study, introduced a novel architectural framework for aggregated subscriber profile solution, which provides a dynamic aggregation of subscriber profile derived from various identified data sources.

\section{AGGREGATED SUBSCRIBER PROFILE}

Subscriber Data in a CSP network has been proliferated across several network elements such as HSS, HLR, CRM, LDAP, Relational Databases, Billing System and is operational across many applications and services. The increased demand for Convergent Telecom Application and Services is a challenge for CSPs, as to address the data integration needs between applications and data sources and their access data management. The proposed Architectural Framework is an attempt to provide a unified Aggregated Profile derived from various heterogeneous Data Sources. The Proposed Framework provides an effective aggregation of data and exposing unified profile to Convergent Telecom applications and Services. Subscriber's profile data is distributed across various external systems, including Social Network Corporate/Enterprise Data sources. Framework proposed by (Michele et al., 2008) provides flexibility to adopt such external information and helps CSPs to expose meaningful and dynamic Subscriber profile information to the Application Plane. Proposed Framework is based on Service Oriented Architecture and Data Services Principle and Techniques demonstrated by (Subramanyam and Walker, 2009), discussed by (Carey et al., 2012; Manu, 2005).

The proposed Architectural Framework enables Service Providers in achieving better service offerings with faster time to market by minimizing the integration efforts with various data sources and provides efficient data access governance mechanisms to Service Providers. Schematic of the Architecture is as shown in Fig. 1.

\subsection{Subscriber Profile Definition}

Service Providers define their aggregated subscriber profile schema and authorize only specific portions of the same, to various applications based on their needs. 


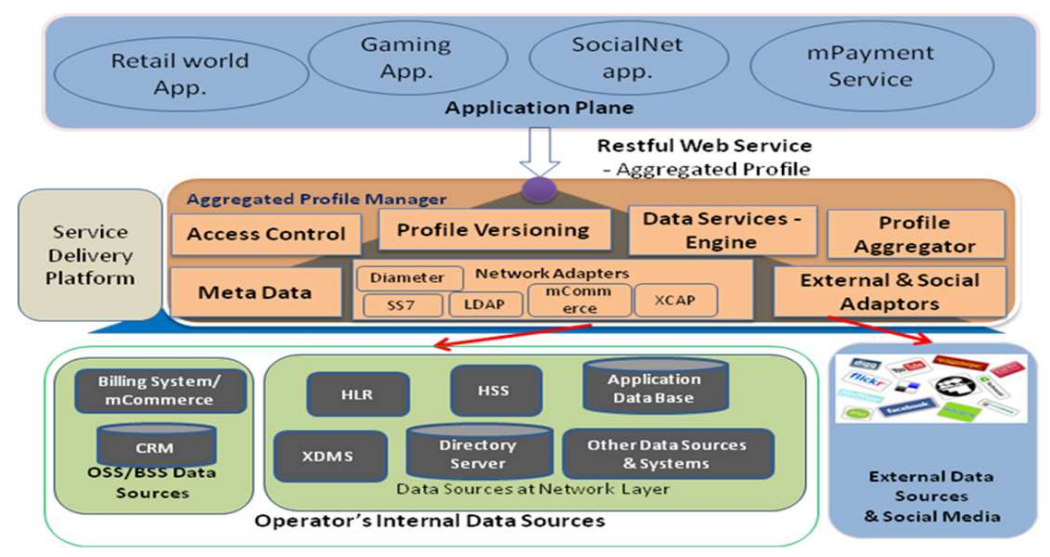

Fig. 1. Schematic of aggregated subscriber profile solution

Profile modeling is done using XML Schema language. During profile definition, different parts of the overall profile information may expect to be constituted from various data sources-both internal and external. These details are captured as a part of Meta Data.

\subsection{Data Sources and Adaptors}

Various typical subscriber data sources (Service Provider's Network layer and BSS/OSS) in any Service provider's environment and the data access interfaces are as tabulated in Table 1. These interfaces are based on 3GPP standards. For accessing these data elements Java based Adaptors built using appropriate integration points are based on 3GPP, IMS, EPC standards for HSS, HLR/VLR, CRM, OSS/BSS Systems.

\subsection{Subscriber Data at Internet and SocialNet}

Users or Subscribers active profile information is available over Internet and Social Network as a result of subscriber's active participation. For the past 5 years, Internet and Social network usage through Mobiles and Personal Digital equipments (PDAs) are dominating its usage through PCs and Laptops. This is one of the obvious reasons for service providers competing to offer context aware customized services to their subscribers. Majority of popular Internet and Social networking application service providers exposing proprietary APIs or web services interfaces to query the subscriber's profiles. Also most of the next generation internet services, sites integrating their applications with these popular internet and social networking platforms/providers for user's profile information for different requirements such as authentication, subscriber context, behavioral information. (Eg. 50000+web sites integrated with Facebook). Custom adaptors for various external and social media networks developed and deployed as physical data service access points.

\subsection{Data Services-Engine}

Based on the specific profile modeling requirements, physical and logical data services are created and deployed on Data Services Middleware. Data Services Engine as shown in Fig. 2 executes these custom data services and constructs the XML messages and handovers to Profile Aggregator for further processing. Data Services Engine is based on the Data Oriented/Service and Service Oriented Architectural principles as demonstrated by (Subramanyam and Walker, 2009), discussed by (Carey et al., 2012; Manu, 2005).

\subsection{Profile Aggregator Component}

Profile Aggregator performs necessary processing and constructs the subscriber profile as per the Aggregated Profile schema. Aggregator component is responsible for performing different logical relations and constructing the virtual or aggregated profile. Pre and Post processing logics executed on the profile information fetched from various underlying physical or external data sources or interfaces.

Profile Aggregator performs the key operations such as:

- $\quad$ Relates and Constructs aggregated profile by fetching different data from various physical data sources

- Performs preprocessing and post processing on data as per the aggregated profile policies and logical relations

- Filters and presents only authorized or context specific profile view to the requester

- Provides Aggregated profile data as an XML Data as per the Profile Definition

- Exposes as a web service interface to the Applications of Service Provider's Application plane 
Table 1. Typical network data elements of CSPs

\begin{tabular}{|c|c|c|c|}
\hline \# & Data source & Description & Integration mechanism \\
\hline 1 & HLR & $\begin{array}{l}\text { HLR contains pertinent user information, } \\
\text { including account status and preferences, ad-dress }\end{array}$ & $\begin{array}{l}\text { SS7 (IS41/MAP) or proprietary } \\
\text { inter-face/adaptors }\end{array}$ \\
\hline 2 & HSS & $\begin{array}{l}\text { HSS (for Home Sub-scriber Server) is a database that } \\
\text { contains user-related and sub-scriber-related information. } \\
\text { It also provides support functions in mobility } \\
\text { management, call and session setup, user authentication } \\
\text { and access authorization } \\
\text { It is based on the pre-3GPP Release 4-Home Location } \\
\text { Register (HLR) and Authen-tication Centre (AuC) }\end{array}$ & $\begin{array}{l}\text { SIP/Diameter, also proprietary } \\
\text { interfaces }\end{array}$ \\
\hline 3 & CRM & $\begin{array}{l}\text { Contains subscriber's personal information, such as } \\
\text { contact ad-dresses and phone numbers, location and } \\
\text { other demographic information, purchase information, } \\
\text { service calls, customer support needs and even customer } \\
\text { interaction can be placed in a CRM data base }\end{array}$ & $\begin{array}{l}\text { Web services or proprietary } \\
\text { Interface/API or SQL }\end{array}$ \\
\hline 4 & LDAP server & Enterprise subscriber's details & LDAP protocol \\
\hline 5 & Billing systems & Subscriber's billing and rating information & $\begin{array}{l}\text { Radius, Diameter, mCommerce } \\
\text { Interface, Proprietary APIs }\end{array}$ \\
\hline 6 & OSS systems & Subscriber's quality of service, configuration details & \\
\hline 7 & XDMS & $\begin{array}{l}\text { Web based subscriber's address book and } \\
\text { instant messaging groups }\end{array}$ & $\begin{array}{l}\text { XCAP, SIP also XML } \\
\text { Over HTTP }\end{array}$ \\
\hline 8 & Others & Standard network elements and data sources & $\begin{array}{l}\text { Standard or proprietary } \\
\text { Integration points }\end{array}$ \\
\hline 9 & Relational data-base & Subscribers or users data & SQL \\
\hline
\end{tabular}

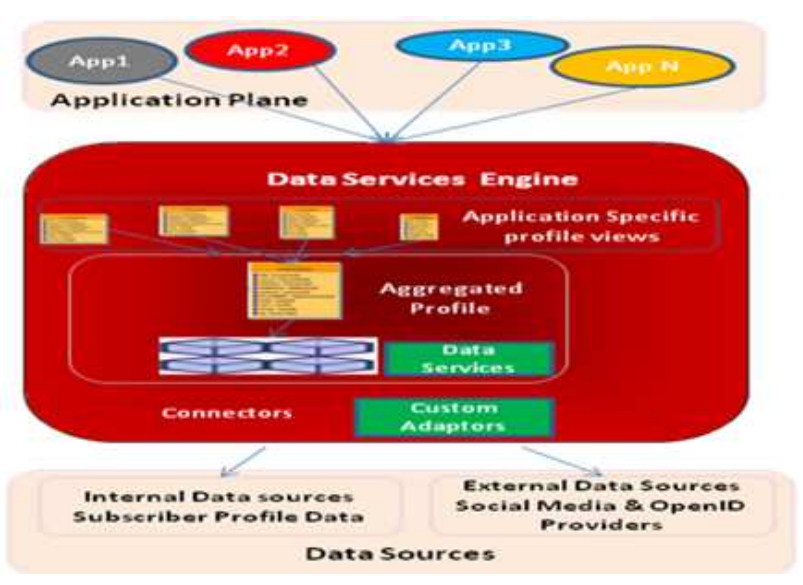

Fig. 2. Data Services Engine

Subscriber profile is constructed through three different phases in-sequence.

\section{Pre-Processing Logic:}

- $\quad$ Read the profile data query request

- Identify the data elements that are required based on input XPATH key

- Perform Meta data lookup and identify the data sources names and generate the same as an ID Tree

\section{Processing Logic:}

- Invoke the Logical data sets parallel and fetch the corresponding Data elements as defined

\section{Post-Processing Logic:}

- Generate final $\mathrm{xml}$ response from the individual data elements received

- Enforce any post-processing rules

\subsection{Meta Data}

Information about Various solutions data access provisioning information, Profile Modeling schemas, Data sources specific access information and other solution specific data maintained as a part of Meta data which may be maintained as XMLs, data base tables.

This includes:

- $\quad$ Aggregated Subscriber Profile Schema (XSD)

- Data sources specific access information

- Logical Relationships among data elements of different physical data sources

- Configuration XMLs of Pre-Processing and postprocessing parameters of profile aggregator 


\section{IMPLEMENTATION APPROACH}

Profile Aggregator Component implemented using J2EE EJB Stateless Session Beans which accepts and returns XML. This component wrapped up with a Java Servlet component which exposes XML over HTTP service as well a Web Service Interface to Application Plane with profile read/search operations. The input XML expected to have an Selection Item with appropriate ID value as shown in Fig. 3. Profile read request intercepted first by configured Pre-Processing logic if any, which is implemented using POJO. ID Tree generator logic of Profile Aggregator component prepares ID Tree XML for the given input parameters as per the Aggregated Profile Model (schema). ID Tree XML contains filled with appropriate markups of the Data Sources, Data Service interface details as shown in Fig. 4. Profile Aggregator Component invokes corresponding Data Services on the Data Service Engine and prepares the Final XML. In this process, ID Tree XML acts as skeleton and the final $\mathrm{XML}$ is been filled with respective XML responses generated from Data Services Engine. Post processing logic if any configured, applied on the generated XML and further final XML Profile response as shown in Fig. 5, returned back to the Profile Requester.

\subsection{Typical Aggregated Data Model Definition}

Aggregated Data model vary for Service Providers. Defined a typical Data Model for proof of concept implementation purpose and considered couple of data sources using Relational Data Base tables. Implemented physical and logical Data services definitions using Data Services Engine as demonstrated by (Subramanyam and Walker, 2009), discussed by (Carey et al., 2012; Manu, 2005). Aggregated Profile definition considered with elements as stated below:

PartyProfile is the root element and it contains an Individual or an Organization sub-elements

Individual Element Contains:

- Zero or more Identification element which represents all the human readable identifications of a person like MSISDN, Email ID

- Zero or more User element which represents the Individual as a user of Service Provider's services

- Zero or more Customer element which represents the Individual as a Customer of SP having a billing relationship

User Element Contains:
- Zero or more Identification element which represents all the human readable identifications of a person like MSISDN, Email ID

- Service classification which identifies the service that the subscriber is using

Customer Element Contains:

- Zero or more Identification element which represents all the human readable identifications of a person like MSISDN, Email ID

- Zero or more Customer Acccount elements which represent the billable accounts that are related to the products that are purchased by the customer of Service Provider

Customer Account Element Contains:

- Zero or more Identification element which represents all the human readable identifications of a person viz. MSISDN, Email ID

\subsection{Aggregated Profile Processing Results}

In our Proof of Concept implementation, we considered two data sources of independent RDBMS instances. Created Physical and logical data services to fetch respective data chunks. Populated ID_TREE table with the Unique ID values of each physical data source with appropriate pointers to its data source. Input query XML contains mainly Select Item ID, Filter criteria (optional).

Preprocessing logic of Profile Aggregator, prepares ID Tree and invokes each logical data services on Data Service Engine. XML Response prepared as per the ID Tree XML and post processing logic applies the filter criteria and prepared final output XML. Samples XMLs captured as below.

\subsection{Summary of Performance Results}

Initial performance details measured based on Proof of Concept implementation. Further performance tuning techniques applied to achieve better performance results. Performed multiple analyses and stage wise, applied performance tuning techniques and finally achieved reasonable performance metrics. At every stage profiling tools used for analysis and by following the process of identification by elimination as discussed by (Chen and $\mathrm{Su}, 1995)$, arrived with 100 TPS with each response has 100 profile objects (xml elements).

\subsection{Rollout Process for CSPs-Discussion}

Different Communication Service Providers may have multiple data sources that support standard interfaces or proprietary APIs and interfaces. 


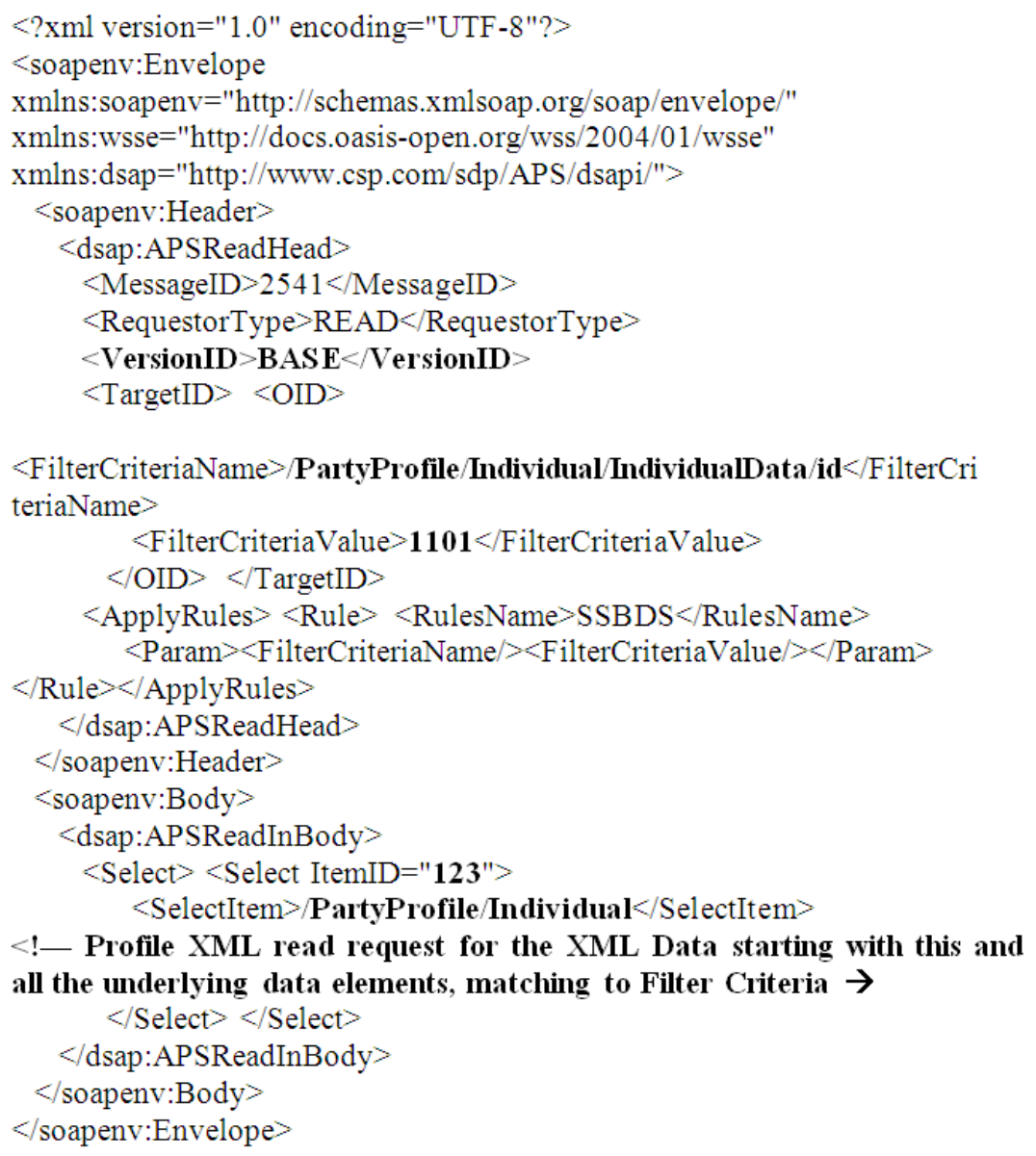

$<$ FilterCriteriaName $>$ PartyProfile/Individual/IndividualData/id $<$ FilterCri teriaName $>$

$<$ FilterCriteriaValue $>\mathbf{1 1 0 1}<$ FilterCriteriaValue $>$

$<$ OID $><$ TargetID $>$

$<$ ApplyRules $><$ Rule $><$ RulesName $>$ SSBDS $<$ RulesName $>$

$<$ Param $><$ FilterCriteriaName $/><$ FilterCriteriaValue $/><$ Param $>$

$</$ Rule $><$ ApplyRules $>$

$<$ dsap:APSReadHead $>$

$</$ soapenv:Header $>$

$<$ soapenv:Body>

$<$ dsap:APSReadInBody $>$

$<$ Select $><$ Select ItemID="123" $>$

$<$ SelectItem $>$ /PartyProfile/Individual $<$ SelectItem $>$

$<$ ! - Profile XML read request for the XML Data starting with this and all the underlying data elements, matching to Filter Criteria $\rightarrow$ $<$ Select $></$ Select $>$

$<$ dsap:APSReadInBody $>$

$<$ soapenv:Body $>$

$</$ soapenv:Envelope $>$

Fig. 3. Input request XML message to aggregated profile solution

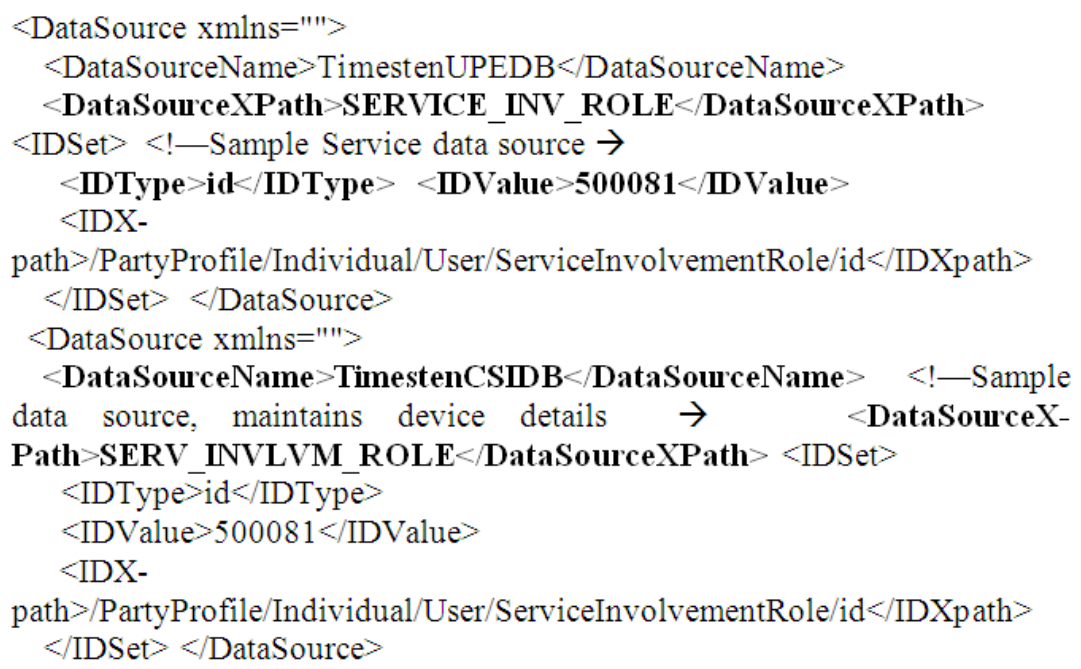

Fig. 4. Identified data source (ID Tree) XML generated by pre-processing logic 


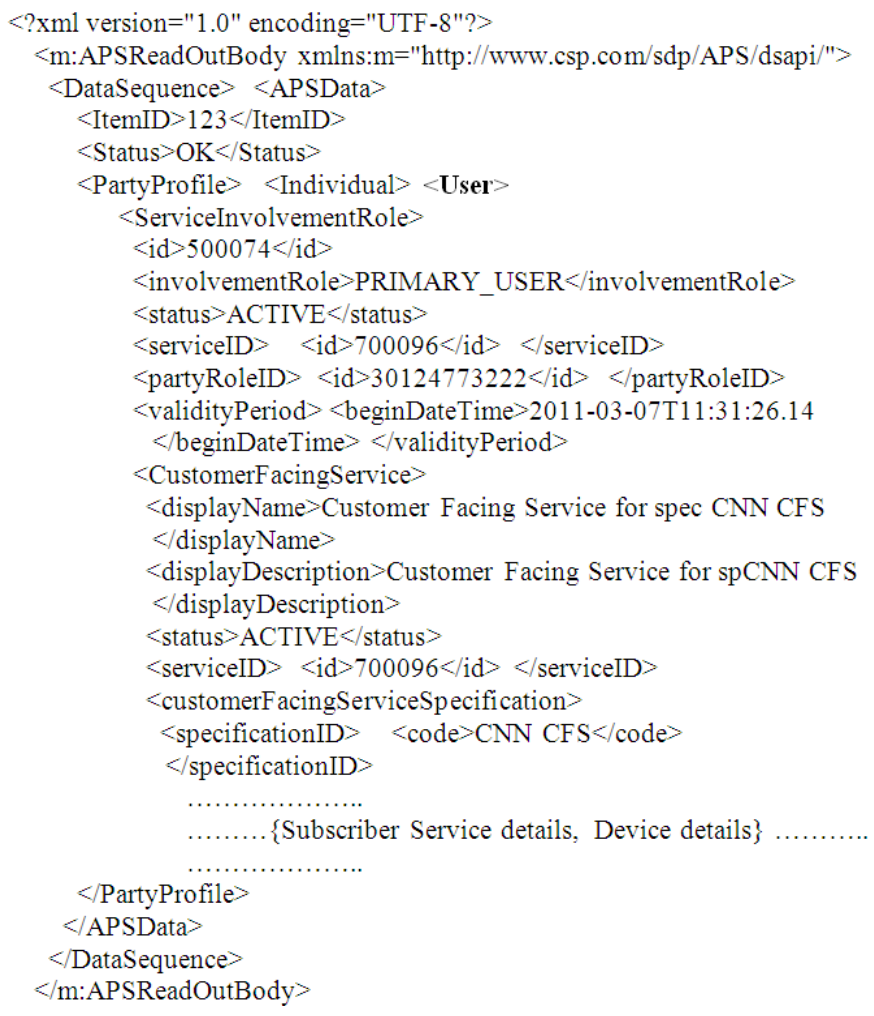

Fig. 5. Subscriber profile query response

CSPs can define a common aggregated profile definition and the identified physical data sources. Aggregated Profile Solution rolled out for a CSP involves different high level steps as below:

Step1: Identify and define the Aggregated Profile View(s) definition using XML Schema corresponding to existing data structure/models.

Step2: Identify the Physical Data sources and the logical relationships through identified relationships among data elements of multiple data sources.

Step3: Define Physical data services to fetch specific data elements from corresponding data sources.

Step4: Additional customizations in the solutions achieved through minimal configurations.

Performance results illustrated in previous sections were based on Proof of Concept implementation. However performance results may vary based on number of Profile Elements in a given aggregated profile definition which essentially defines the throughput, Number of Network Data Sources/Elements from which Aggregated Profile derived, response time of each data sources. Hence performance analysis and tuning needs to be analyzed case to case basis as a part of solution rollout process.

\section{CONCLUSION}

With the proliferation of convergent services and applications, managing and controlling their integration needs with various internal and external data sources is going to be a challenge. The proposed Aggregated Profile Management Solution deployed in Service Provider's Network enables better data access governance and intenerated with any Service Delivery Infrastructure including architectural frameworks proposed by (Dulipala and Ramachandran, 2009).

Light weight Data Access Profile (LDAP) is widely used protocol for accessing user data. Our current focus is towards identifying technical approaches to expose Aggregated Profile through LDAP protocol. Also our future work focused towards better performance results. 


\section{REFERENCES}

Adzic, J., V. Fiore and S. Spelta, 2001. Data warehouse population platform. Databases Telecommun., 2209: 9-18 DOI: 10.1007/3-540-45432-2_2

Carey, M.J., N. Onose and M. Petropoulos, 2012. Data services. ACM Commun., 55: 86-97. DOI: $10.1145 / 2184319.2184340$

Chan, J., 2004. Building data warehouses using the enterprise modeling framework. Proceeding of the 9th Americas Conference on Information Systems, Aug. 4-6, Tampa, FL, USA.

Chan, J.O., 2005. Toward a unified view of customer relationship management. J. Am. Acad. Bus., 6: 3232

Chen, Y.H. and S.Y.W. Su, 1995. Identification- and elimination-based parallel query processing techniques for object-oriented databases. J. Parallel Distrib. Comput., 28: 130-148. DOI: 10.1006/jpdc.1995.1095

Dulipala, J . and D.V. Ramachandran, 2009. SCA for context-aware mobile applications. Int. J. Recent Trends Eng., 2: 235-239

Ericsson, 2011. User Profile Gateway (UPG), Centralized User Database (CUDB) Product/Solutions Overview.

Karvell, L ., J. Marconi, R. Kumar, M. Borkenhagen and D. Law et al., 2011. User profile aggregation. U.S. Patent.
Lucent, A., 2011. Is there a Role for Communication Service Providers in Social Media? Strategic White Paper, Alcatel Lucent Press.

Manu, M.R., 2005. Information integration using the aqualogic data services platform. ORACLE

Michele, M., D. Palmisano, F. Zani and S. Tripodi, 2008. Towards an OpenID-based solution to the Social Network Interoperability problem. Proceedings of the W3C Workshop on Future Social Netwo, (SN' 2008), FInES.

Mottishaw, P., 2009. Subscriber data management outlook, OSS observer-research report. Analysys Mason Ltd.

Ohnishi, H., Y. Yamato, M. Kaneko, T. Moriya and M. Hirano et al., 2007. Service delivery platform for telecom-enterprise-internet combined services. Proceedings of the IEEE Global Telecommunications Conference, IEEE Xplore Press, Washington, DC., pp: 108-112. DOI: 10.1109/GLOCOM.2007.28

Perry, J.R., K.D. Snow and D. Black, 2006. Distributed user management information in telecommunications networks. U.S. Patent.

Subramanyam, M. and M. Walker, 2009. JBoss enterprise data services platform in the enterprise. World.

Yuhong, C., 2011. Exploring subscriber data management for MBB. HUAWEI. 